Review

\title{
Biological databases and tools for neurological disorders
}

Computational approaches to study of neuronal impairment is rapidly evolving, as experiments and intuition alone do not explain the complexity of the brain system. An overwhelming increase in the amount of new data from both theory and computational modeling necessitate the development of databases and tools for analysis, visualization and interpretation of neuroscience data. To ensure the sustainability of this development, consistent update and training of young professionals is imperative. For this purpose, relevant articles, chapters, and modules are essential to keep abreast of developments. This review seeks to outline the biological databases and an- alytical tools along with their applications. It is envisaged that such knowledge could provide a "training recipe" for young scientists and a guide for professionals and researchers in neuroscience.

Keywords

Neurological disorders (NDs); Database; Tools; Neuroinformatics; NeuroDNet; PubMed; Cytoscape 


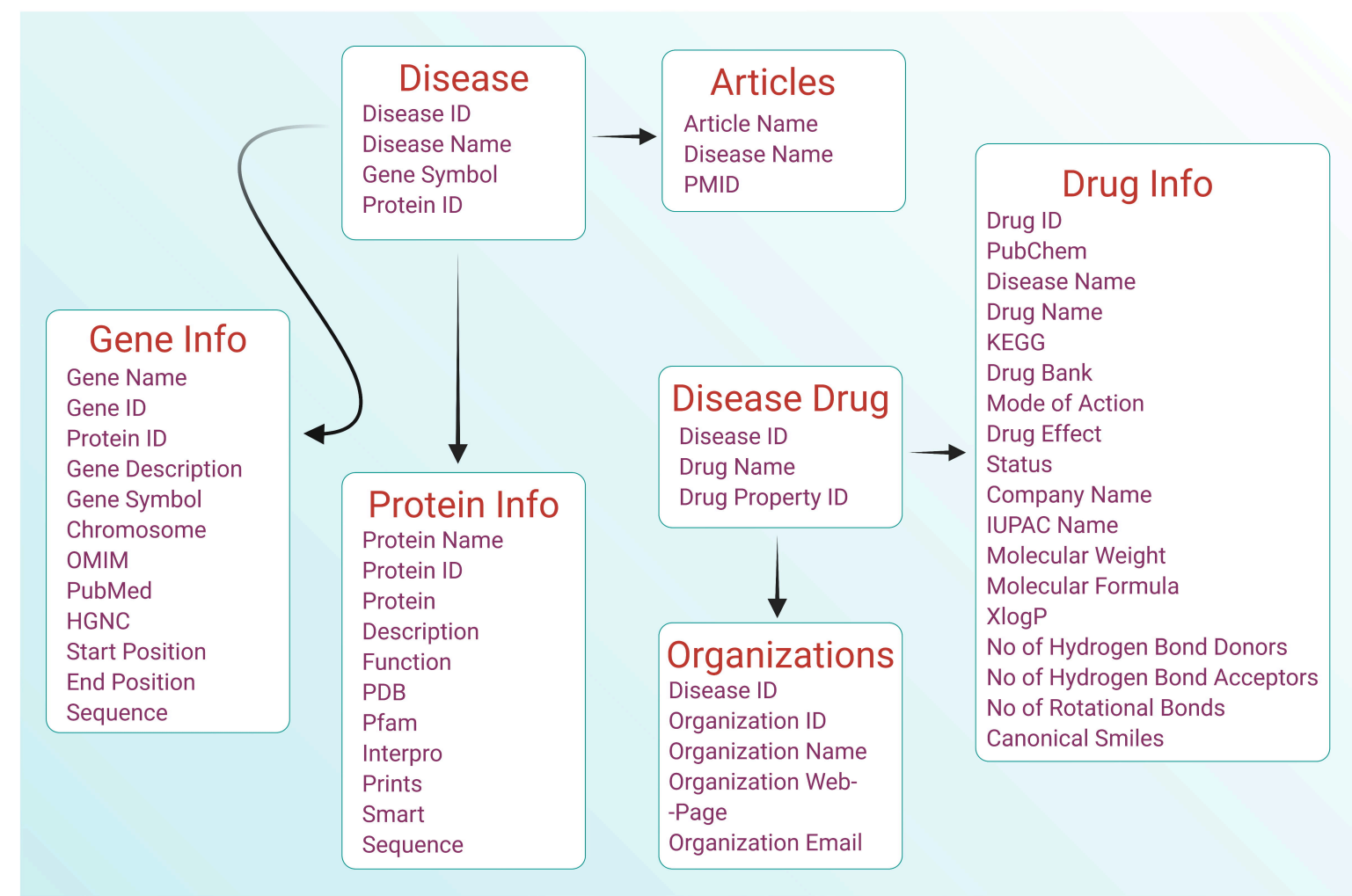

Fig. 1. Schematic representation of various NDs-associated informations which can be extracted using biological database.

\section{Introduction}

Neuronal impairments or neurological disorders (NDs) continue to attract much research attention owing to both their unknown etiology and the interwoven complexities of their underlying molecular and signaling pathways that often make the identification of practical classifications and therapeutic targets difficult. However, with recent advancement in computational methods, investigators are able to employ several databases and tools to demystify these complex networks for the identification of better therapeutic or mechanistic targets $[1,2]$. These resources are not only useful for the study of bio-molecular interaction networks, but also help in network analysis, visualization and the establishment of relationships at the level of their genes and protein products. Databases serve as repositories for the retrieval of gene or protein interaction data, while the computational tools help withn data visualization, the establishment of interaction networks and data analysis. The various forms of, edical interaction networks being analyzed include: gene interaction (GI), protein-protein interaction (PPI), protein-DNA interactions, and protein-RNA interactions [3, 4]. Computational analysis of these interactions has revolutionized the modern understanding of NDs and provides better prospects for drug discovery.

Use of computational a approach to the study of NDs constitutes the field of Neuroinformatics. Recently, research in Neuroinformatics has gained ground, as it appears to be more promising in providing understanding of neural networks and disease. Research in Neuroinformatics extends to theory and methodology, including analytical tools, database design, meta-analysis, data sharing and discussions on computational modeling. The pool of data is vast and heterogeneous in the field of experimental neuroscience and the integration and analysis of these increasingly large-volume, high-dimensional data sets require Neuroinformatics to further understanding of the nervous system [5-8]. Neuroinformatics is at the crossroads of neuroscience and information science [9-11]. It involves the study of the nervous system, its structure, function and diseases such as Alzheimer's disease ( $\mathrm{AD})$, Parkinson's disease (PD), Huntington's disease (HD), Amyotrophic lateral sclerosis (ALS), Prion disease amongst others. Research in neuroscience contains many different sub-disciplines that analyze data from multiple levels of the brain and information science is concerned with the collection, classification, and dissemination of information [12-14]. To solve increasingly complex problems, clinicians and research scientists use computational tools, mathematical models and Neuroinformatic databases provided by neuroinformaticians to collaborate, share information and quantitatively support working theories [15].

\section{The link between computational neuroscience and Neuroinformatics}

Computational neuroscience employs theoretical analysis, mathematical models and abstractions of the brain to un- 


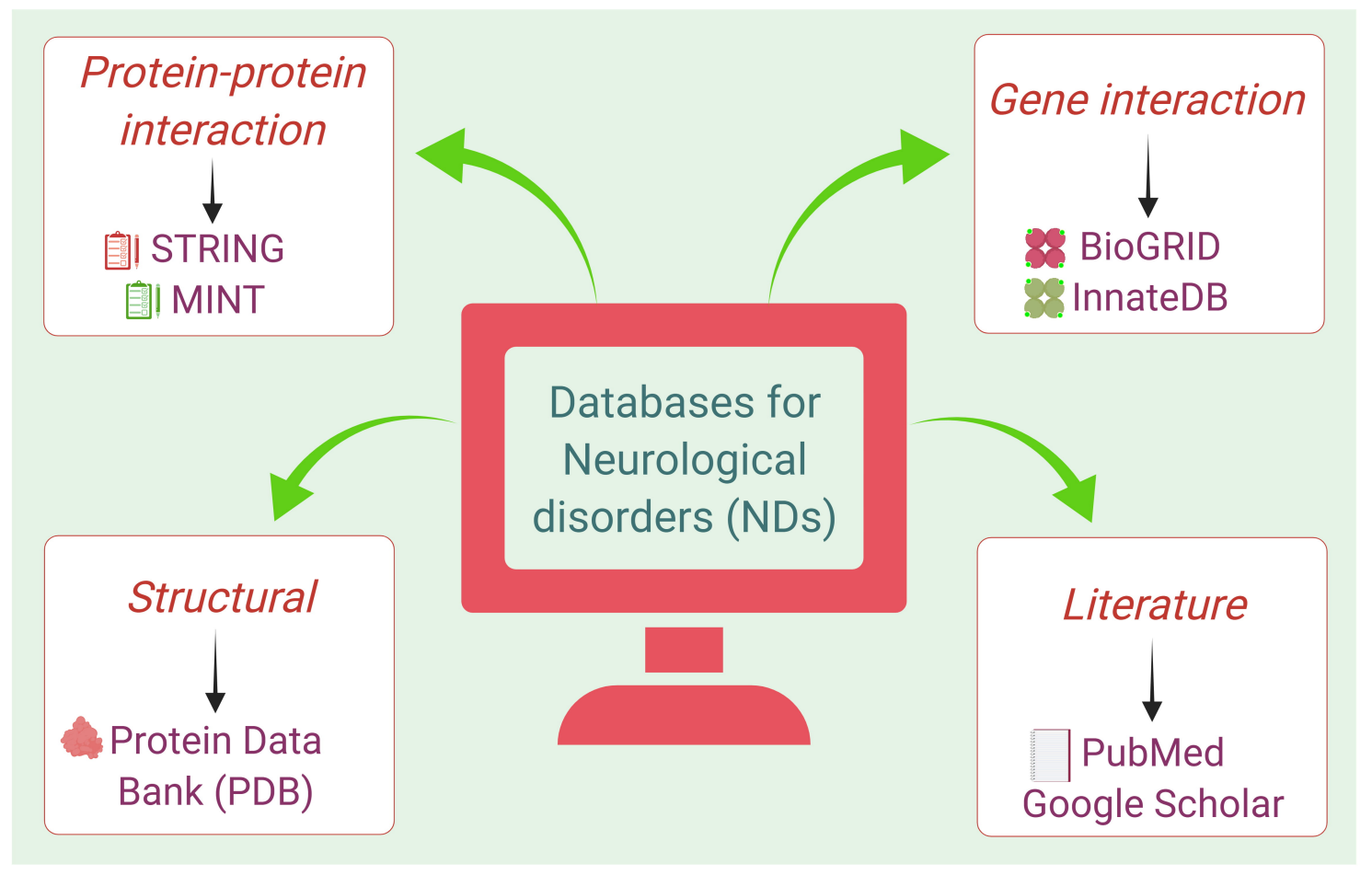

Fig. 2. Schematic classification of in-silico databases for NDs.

derstand the principles that govern the development, structure, physiology, information processing and cognitive abilities of the nervous system. Computational neuroscientists employ mathematical and computational approaches to understand how the brain processes information and informatics helps accelerate this neuroscientific discovery. Neuroinformatics tools such as image analysis, computer simulation and database integration help facilitate neural modeling and collaboration in the field of computational neuroscience. The nature of this sub-discipline of neuroscience is primarily quantitative, so Neuroinformatics is beneficial in the organization and analysis of data derived from computational research. Computational neuroscience differs in more qualitative experiments such as finding the optimal design of nervous systems or exploring various neural connectivity schemes in model networks $[16,17]$.

\section{Fields related to Neuroinformatics}

Neuroinformatics is rapidly evolving due to manifold ways of applying information technology to demystify complex problems related to neuroscience. These ways include development of databases and tools necessary for the analysis, modeling, management, and sharing of neuroscience data (Fig. 1). Thus, Neuroinformatics is multidisciplinary, with each discipline having a reasonable contributory role. Hence, fields related to Neuroinformatics are summarized as; (a) Psychology: Cognitive development and information processing theory, (b) Computer Science: Natural and Bioinspired computation, (c) Philosophy: Computational theory of mind, (d) Engineering: Brain-Computer Interface technology, (e) Medicine: Aging and mental diseases like depression and anxiety, (f) Physical Sciences: Physical processes within neural cells and neuronal networks, (g) Mathematics: Statistical algorithms and Quantifying neuronal differentiation, (h) Chemistry: Molecular structures and their interactions in nervous system, and (i) Biology: Chemical processes and molecular structure.

\section{In silico databases for neurological disorders}

With an increasing number of large databases, data sharing in neuroscience may gradually become as common and useful as it is in genomics, where the existence of very large bodies of data is leading to increased knowledge as well as products and services linked to the improvement of human health. Numerous databases are being employed to study NDs; these databases can be open source (freely accessible) or proprietary (commercially available). Regardless of the source, databases for the study of NDs can be broadly divided into four as shown in Fig. 2.

Further, some of these databases along with their roles in the study of neuronal impairment are enumerated in Table 1 (Ref. [3, 4, 18-37]), while the commonly used ones are discussed below.

\subsection{Google scholar}

Google scholar is a web-based platform that enables the search of scholarly literature such as books, reports, and articles present in online libraries or databases. The powerful search engine indexes full-text journal articles, selected web 
pages and other documents. Moreover, it avails users of opportunity to search across various disciplines and establish personal collection of articles via the save button. Interestingly, Google scholar can now efficiently be used to identify the highly-cited paper [38]. Thus, articles pertinent to NDs may also be searched, sorted, and pooled to make a collection for reasonable analysis [39].

\subsection{Protein data bank}

Protein Data Bank (PDB) is a freely available structural database that gives the $3 \mathrm{D}$ shape of macromolecules (such as proteins and nucleic acids). It's a primary database whose results are derived from experimental data obtained through $\mathrm{X}$-ray crystallography and NMR spectroscopy. PDB is managed by Worldwide Protein Data Bank (wwPDB) and the current entries are 165,117 . It affords users the opportunity of searching and downloading 3D structures and their visualization. PDB helps in the study of NDs by providing 3D shapes of several proteins involved, their molecular bonds, fragments, and domains which enable researchers to study the protein for modeling, ligand interactions, and drug design [31-33].

\subsection{PubMed}

PubMed is an open-source literature database being overseen by the US National Library of Medicine. PubMed as a gateway to MEDLINE database, contains freely available research papers, abstracts, older and recent references, PubMed Central (PMC) citations among others. As of June 2021, PubMed database has over 32 million citations and abstract of biomedical research. In the study of NDs, PubMed is utilized for the construction of datasets through literature search, effective searching is achieved by using keywords relevant to the NDs and related articles are collected. The database can also be used to confirm data extracted from other sources [22].

\subsection{Universal protein resource}

Universal Protein Resource (UniProt) is a freely accessible repository for annotated protein sequences; with unique protein ID, gene name, and gene symbols. UniProt is a combination of Swiss-Prot, Translated European Molecular Biology Laboratory (TrEMBL) and International Protein Sequence Database (PIR-PSD). UniProt has over 564,638 sequence entries, consisting of about 204 million amino acids from 278,054 references as of April 2020. Researchers make use of UniProt in studying neuronal impairments through retrieval of unique protein ID, gene names and symbols for construction and analysis of NDs complex networks, and extraction of specific protein sequence for further analysis $[4,24,29]$.

\subsection{Search tool for the retrieval of interacting genes/proteins}

Search Tool for the Retrieval of Interacting Genes/Proteins (STRING) is an open-source web-based repository of over 2,000 million predicted and known protein interactions. STRING database contains PPI networks based on functional associations and allows users to establish a relationship between interacting proteins/genes through the confidence score. PPI in the STRING database is obtained from literature curation of experimentally determined interactions. Users can search for a gene/protein in the database through a Uniprot ID, single or multiple protein names, amino acid sequence (single or multiple). Nodes represent proteins or genes, while edges in different colors indicate the method used in detecting the relationship, confidence score is used for predicted functional associations [40]. In studying NDs, STRING is employed for the establishment of PPI networks, determination of confidence or evidence level for each interaction, gene ontology analysis, Reactome pathways, among others [3, 26].

\subsection{Biological general repository for interaction datasets}

Biological General Repository for Interaction Datasets (BioGRID) is a freely accessible database of genetic and PPI networks. It also contains post-translational modifications and chemical interaction data. In the current version 3.5.186, detailed information includes $1,871,024$ protein and genetic interactions, 28,093 chemical associations, and 874,796 posttranslational modifications obtained from humans and major model organisms. BioGRID provides gene and PPI data necessary for the study of neuronal impairment, the data extracted is used to construct datasets for network analysis in NDs [30].

\subsection{Other databases}

Web of science: it is a subscription-based website published by Thompson Reuters. Web of science, being an interdisciplinary database, offers access to various databases with comprehensive citation record such as Social Sciences Citation Index (SSCI) and Science Citation Index Expanded (SCIEXPANDED). The SSCI contains record from Psychiatry, Psychology, and public health, while SCI-EXPANDED covers the area of medical discipline [41]. The website may be used to access various articles relevant to NDs.

In addition to the above databases, several others have been reported. For instance, the Database of Neurodegenerative Disorders (DND) provides a user-friendly interface and is designed as a source to improve research on NDs. It is developed as an open-source software system using Mysql5.0.18-Win32 and PHP-5.2.0 and uses the relational data model (Gowthaman et al., 2007 [42]). Further, a schematic diagram addressing the DND is shown in Fig. 1.

Similarly, another database known as NeuroGeM has recently been developed. NeuroGeM integrates a comprehensive collection of literature data on genetic modifiers of NDs and associated genetic information from an array of databases. To give complete information on genetic modifiers, NeuroGeM also integrates information from genome databases (SGD, FlyBase, EBML, WormBase, HGNC, and MGI), the homologous gene databases (HomoloGene and InParanoid), protein interaction database (STRING), and Gene Ontology [43]. Likewise, NDDVD, an integrated and manually curated Neurodegenerative Diseases Variation Database 


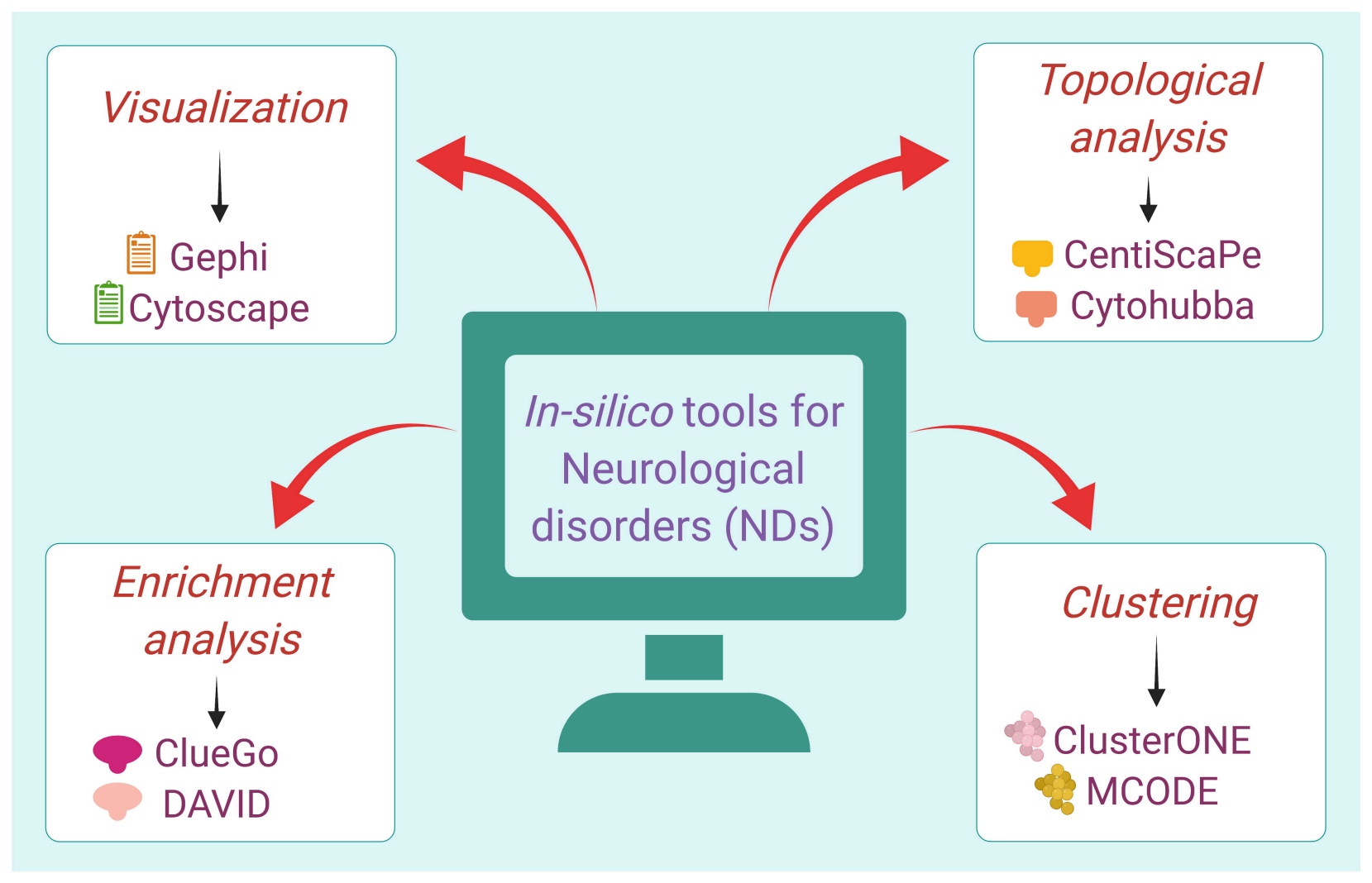

Fig. 3. Schematic classification of in-silico tools for NDs.

has recently been developed. NDDVD can provide data for the identification of common variation spectrum within Neurodegenerative disease (NDDs) and for developing universal biomarkers or drugs for NDDs as well. It can also serve as a research platform for further discovering relationships between diseases and genetic variations. The integrated database will act as a precious tool for quickly querying the NDD-related variations and systematically analyzing the relationships between diseases and variations [1].

\section{In silico tools/software for neurological disorders}

Many tools have been developed to study NDs; these can be freely accessible or commercially available.

Some Neuroinformatics tools are meant for analysis, modelling, and visualization of neural systems such as Cytoscape, Gephi and EEGNET. Others are simply plug-ins that provide interface for various analysis such as ClusterONE and CytoHubba, while others were developed for structuring data to create consistent terminology and formats for the sharing of data such as BIRN (Biomedical Informatics |Research Network). In silico tools for the study of NDs can be divided into four broad categories as shown in Fig. 3, while Table 2 (Ref. $[3,23,24,26,34,44-54])$ summarizes some of them. Further, the commonly used tools are discussed below.

\subsection{Cytoscape}

It is an open-source software developed by the Institute for Systems Biology for visualization and analysis of biomolecular interaction networks. Cytoscape works with almost all operating systems and provides a platform for data integration, gene and PPI analysis, and visualization. The presence of several layout algorithms makes the software robust for network construction, while several plug-ins (which are freely available in the Cytoscape App store) enhances its usability in several processes such as network and molecular profiling analyses, connection with databases, different layout, and scripting [40]. Moreover, many other analyses are possible through the Cytoscape App such as topological analysis, functional enrichment analysis, clustering, etc. Cytoscape is a very important software for the study of NDs owing to its versatility in modeling, visualization, and analysis. Through this great software, complex networks in NDs are visualized and analyzed for several purposes such as overlapping of pathways in NDs, molecular interactions, functional enrichment, and topological analysis [34, 44].

\subsection{EEGNET (Electroencephalography NET)}

Is a recently developed flexible and user-friendly tool. The open source in silico tool, running under MATLAB, allows the visualization and analysis of connectome from Magneto/Electroencephalography (M/EEG) recordings. 
Table 1. List of in silico databases for NDs.

\begin{tabular}{|c|c|c|c|}
\hline Database & Description & Use in NDs & Reference \\
\hline NeuroDNet & Database for analysis and construction of ND networks & $\begin{array}{l}\text { Retrieval of gene and proteins related to NDs and their } \\
\text { interactions for analysis }\end{array}$ & [18] \\
\hline Alzheimer's Disease Neuroimaging Initiative (ADNI) & ADNI database has information on $\mathrm{AD}$ for monitoring of research and treatments & Mining of data relevant to $\mathrm{AD}$ for further research & [19] \\
\hline National Alzheimer's Coordinating Center (NACC) & $\begin{array}{l}\text { NACC database contains collection of information on } \mathrm{AD} \text { patients from various } \\
\mathrm{AD} \text { centers }\end{array}$ & $\begin{array}{l}\text { Extraction of data of } \mathrm{AD} \text { for analysis, monitoring and } \\
\text { research }\end{array}$ & [20] \\
\hline Online Mendelian Inheritance in Man (OMIM) & OMIM is a database of human genes and genetic disorders & Acquisition of data for disease genes in NDs & [21] \\
\hline PubMed & Free online database for literature search & $\begin{array}{l}\text { Construction of dataset for NDs through literature } \\
\text { search }\end{array}$ & [22] \\
\hline Interologous Interaction Database (I2d) & I2d contains protein interaction networks data & Construction of ND protein interaction networks & [23] \\
\hline Universal protein resource (Uniprot) & $\begin{array}{l}\text { Uniprot database is a collection of proteins with their unique IDs, gene name, gene } \\
\text { symbol and sequences }\end{array}$ & $\begin{array}{l}\text { Retrieval of unique protein ID, symbols or sequence for } \\
\text { ND proteins }\end{array}$ & {$[4,24]$} \\
\hline $\begin{array}{l}\text { Kyoto Encyclopedia of Gene \& Genome (KEGG) path- } \\
\text { way }\end{array}$ & Open source database for pathway mapping & Collection and mapping of ND pathways & {$[22,25]$} \\
\hline $\begin{array}{l}\text { Search Tool for the Retrieval of Interacting } \\
\text { Genes/Proteins (STRING) }\end{array}$ & STRING is a database of PPI network and gene ontology analysis & $\begin{array}{l}\text { Establishment and growing of PPI networks for NDs } \\
\text { and also for gene ontology }\end{array}$ & {$[3,26]$} \\
\hline $\begin{array}{l}\text { Online Predicted Human Interaction Database } \\
\text { (OPHID) }\end{array}$ & OPHID is a web-based database of human predicted PPI & For making interaction dataset of NDs & [27] \\
\hline Human Protein Reference Database (HPRD) & HPRD is PPI database with disease associated to each protein & $\begin{array}{l}\text { Text mining of PPI data and disease related to each pro- } \\
\text { tein in NDs }\end{array}$ & [22] \\
\hline Molecular INTeraction Database (MINT) & MINT is a repository of molecular interactions which are experimentally verified & $\begin{array}{l}\text { For collection of molecular interaction data related to } \\
\text { proteins of NDs }\end{array}$ & [28] \\
\hline Database of Interacting Protein (DIP) & DIP is a collection of experimentally determined PPI & $\begin{array}{l}\text { Text mining and acquisition molecular interactions of } \\
\text { ND proteins }\end{array}$ & [26] \\
\hline InnateDB & Integration of signal pathways and gene interactions of innate immune responses & $\begin{array}{l}\text { Retrieval of gene interactions of innate immune re- } \\
\text { sponses related to NDs }\end{array}$ & [29] \\
\hline $\begin{array}{l}\text { Biological General Repository for Interaction Datasets } \\
\text { (BioGRID) }\end{array}$ & BioGRID contains genetic and PPI data & Data mining for PPI and genetic networks of NDs & [30] \\
\hline Protein Data Bank (PDB) & PDB contains proteins in $3 \mathrm{D}$ format & Retrieval of 3D protein structures of NDs & {$[31-33]$} \\
\hline Genetic Home Reference & Open source website that contains information on human genetic variations & Retrieval of genetic variations data related to NDs & [34] \\
\hline NCBI Gene Expression Omnibus (GEO) & A repository of gene expressions data & $\begin{array}{l}\text { Extraction and construction of ND gene expression } \\
\text { datasets }\end{array}$ & [25] \\
\hline Human Gene Mutation Database (HGMD) & Repository of human inherited gene mutations & Extraction of mutated or inherited genes related to NDs & [30] \\
\hline ALSOD & $\begin{array}{l}\text { Provides more detailed information's about various factors of ALS including, ge- } \\
\text { netic, proteomic, and bioinformatics information associated with the disease }\end{array}$ & Helps in providing information about ALS & [35] \\
\hline
\end{tabular}


Table 2. List of in silico tools for NDs.

\begin{tabular}{|c|c|c|c|}
\hline Tool & Description & Use in NDs & Reference \\
\hline BioCyc Database Colletion & $\begin{array}{l}\text { Consists of } 17832 \text { Pathway/Genome Databases (PGDBs) for model eukaryotes, thousands } \\
\text { of microbes, and tools for analysis }\end{array}$ & Pathway analysis of various NDs & [36] \\
\hline Reactome & $\begin{array}{l}\text { Open access, manually curated pathway database that contains intuitive tools for visualiza- } \\
\text { tion, modelling, interpretation, and analysis of genome and pathways }\end{array}$ & Pathway analysis and gene ontology analysis of NDs & [37] \\
\hline Cytoscape & Open source tool for analysis and visualization of complex networks & Visualization and analysis of complex gene and PPI networks in NDs & {$[34,44]$} \\
\hline DAVID & It is a functional analysis enrichment tool that gives annotation in tabular form & Provide functional annotation of ND genes and their gene ontology analysis & {$[26,45]$} \\
\hline MATLAB & Tool for topological analysis and measurement of centrality & For characterization of ND networks and assessment of centrality & [3] \\
\hline ClueGO & A Cytoscape plug-in for analysis of biological enrichment pathways & Analysis of ND pathways with respect to their gene ontology terms & [23] \\
\hline Pathway Linker & A Cytoscape tool that links proteins to signal pathways & Categorization, visualization and connection of ND proteins to signal pathways & [46] \\
\hline BLAST & NCBI tool for aligning biological sequences & Comparing gene/protein sequences in NDs & [24] \\
\hline FatiGo & Web-based tool for finding gene ontology terms for group of genes & Extraction of gene ontology terms for ND genes in association with other genes & [47] \\
\hline Gephi & Open source tool for network visualization and analysis & Construction of ND networks, visualization and their analysis & [48] \\
\hline ClusterONE & $\begin{array}{l}\text { It's a standalone App or Cytoscape plug-in that clusters proteins and find overlapping ones } \\
\text { in a complex network }\end{array}$ & For identification and clustering of proteins in ND networks & [49] \\
\hline Cytohubba & It's a java Cytoscape plug-in for several topological analyses & For topological analysis of various ND networks & [48] \\
\hline CluePedia & Another tool for clustering networks and analysis of enrichment pathways & Analyzing ND enrichment pathways and clustering networks & [49] \\
\hline Binom & A plug-in of Cytoscape that enhances the manipulation of biological networks & Facilitates manipulation of ND networks & [44] \\
\hline CentiScaPe & A plug-in of Cytoscape that helps in topological analysis such as centrality measurements & Enhances the topological analysis of PPI in ND network & {$[50]$} \\
\hline MetaCore & Commercially available tool for functional analysis of experimental data & For functional analysis of ND data & [51] \\
\hline NetworkAnalyzer & Multi-functional tool in Cytoscape for analyzing topological parameters & Helps in analyzing wide range of topological parameters in ND networks & {$[50]$} \\
\hline $\begin{array}{l}\text { Molecular Complex Detec- } \\
\text { tion (MCODE) }\end{array}$ & A Cytoscape App that identifies highly connecting nodes (clusters) in complex PPI network & Facilitates the Identification of clusters with high quality in PPI networks of NDs & [51] \\
\hline CytoCluster & A platform-independent App for visualization and analysis of biological network clusters & $\begin{array}{l}\text { ND networks are visualized and their network clusters can be analyzed with this } \\
\text { tool }\end{array}$ & [52] \\
\hline BisoGenet & Another Cytoscape plug-in for gene network construction and topological analysis & For ND network construction and topological analysis & [53] \\
\hline ToppFun & $\begin{array}{l}\text { Open source functional enrichment analysis tool that provide annotation features like gene } \\
\text { ontology terms, microRNAs, PPI etc. }\end{array}$ & To annotate the function of microRNAs, PPI, pathways etc. in NDs & [54] \\
\hline
\end{tabular}


The neural networks being analyzed could either be at reconstructed cortical sources level or even at the level of scalp [55].

\subsection{ClusterONE (Clustering with overlapping neighborhood expansion)}

ClusterONE is a graph clustering algorithm that exists as a standalone App or a plug-in in Cytoscape or ProCope. ClusterONE helps in identifying overlapping protein complexes in the PPI network and also generates the desirable clusters. In the study of NDs, it facilitates the finding of protein in interwoven networks and the construction of PPI network clusters [49].

\subsection{Database for annotation, visualization \& integrated discovery (DAVID)}

DAVID is an internet-based functional enrichment analysis tool that gives holistic functional annotations for a large list of genes. The various forms of annotations included are interacting proteins, protein functional domains and motifs, the association between genes and disease, gene ontology terms, and literature. Other features include visualization of pathway maps and inter-conversion of gene identifiers. DAVID is used to provide a list of functional annotations for complex ND genes in tabular form as well as visualization of their pathway maps $[26,45]$.

\subsection{Cytohubba}

Cytohubba is a java plug-in of Cytoscape that provides a user-friendly interface for topological analysis of PPI networks. Cytohubba provides analysis through eleven topological scoring methods to identify essential nodes in the network, it allows the construction of sub-network (or subgraph) for the essential nodes to enhance the further analysis of direct interaction with top-ranked nodes, this facilitated node retrieval function and extraction of user interesting network make Cytohubba better option than other tools like CentiScaPe [56]. Consequently, Cytohubba is used by researchers for topological analysis of various PPI networks in NDs [48]

\subsection{Basic Local Alignment Search Tool (BLAST)}

BLAST is an algorithm for comparing biological sequences developed by National Center for Biotechnology and Information (NCBI). BLAST is an open-source webbased domain, that is compatible with different operating systems and allows researchers to align unknown (or query) sequences of proteins or nucleic acids with reference sequences in target databases to identify regions of similarities and conserved domains. BLAST helps in comparing various unknown protein or gene sequences of NDs with reference sequences to identify conserved domains. It is also used in modeling for unveiling regions of targets by ligands in NDs [24].

\section{Future trends in \\ Neuroinformatics/computational neuroscience}

Advancements and successes made in the field of Neuroinformatics unveil more challenging perspectives. This chal- lenge combines two tasks; development of new tools for understanding of neural networks and diseases on one side, and effective use of those computational tools and approaches on the other. Firstly, brain consists of large number of neurons with high complexity of structural connectivity that changes with experience. In the recent years, there is rapid increase in an overwhelming amount of new data from both theory and computational modeling to further understand the complex dynamic system. The new accruing data cover the area of brain connectomics, transcriptomics and neurophysiology, effective data analysis and interpretation are key to understanding of brain diseases. This challenge does not only necessitate collaborative efforts across the related fields, but also increase the demand for development of novel analysis tools [57].

Secondly, experiments and intuition alone could not explain the complexity of brain system, consequently, modern computational neuroscience is expanding synergistically with experimental research. One of the major benefits of this synergy is the increased use of artificial intelligence (AI) and machine learning (ML) for computational modeling and data analysis in neuroscience [58]. However, the current limitation of ML in recognizing higher cognitive functions and complex network visualization will continue to put pressure on Neuroinfromatics to develop more dynamic tools and methodologies. With the continues influx of an overwhelming amount of computational databases and tools, development of infrastructure, methodologies, and trainings for effective handling of the heterogeneous datasets will constitute the future challenge in Neuroinformatics and neuroscience [2].

Considering the successes recorded in Neuroinformatics, more attention needs to be given to infrastructural development, funding, and training to ensure the sustainability of brain research. Training opportunities would attract young talents that will drive the future development of computational algorithms and analytical tools. It's through regular training that professionals and young talents will be kept abreast of recent development. Therefore, having articles and chapters - like the present one - that summarize recent advances in analytical tools and databases would be a "training recipe".

\section{Conclusions}

Computational approach to study of NDs is rapidly evolving. The increase in synergistic relationship between computational and experimental neuroscience leads to the emergence of more analytical tools and databases. For sustainability of research in neuroscience, development of infrastructure, methodologies, and trainings are necessary. Frequent update and review of the emerging databases and tools along with their functionalities would be a training recipe for young professionals and guide for researchers. This is in a bid to further understand the fundamentals of NDs, their unknown etiology, interwoven complex molecular and signaling path- 
ways. Additionally, it will also help in the development of the drug and further improves the neurotherapeutics. Further study is recommended to explore more of such databases and tools for their pertinent role in Neurological studies.

\section{Author contributions}

NKJ, MBU and AA conceptualized, wrote, and edited the manuscript. SKJ, DKC, GG, SKS, SR, NK, FAK, HD and VU performed the literature survey, drafted and edited the manuscript. NKJ ideated the scheme, performed artwork. PP, KKK, GMA, SO, KD, FZ helped in revision and edited the manuscript. All authors contributed to the article and approved the submitted version.

\section{Ethics approval and consent to participate \\ Not applicable.}

\section{Acknowledgment}

We would like to thanks the senior management of Sharda University for their constant support and encouragement.

\section{Funding}

This research received no external funding.

\section{Conflict of interest}

The authors declare no conflict of interest.

\section{References}

[1] Yang Y, Xu C, Liu X, Xu C, Zhang Y, Shen L, et al. NDDVD: an integrated and manually curated Neurodegenerative Diseases Variation Database. Database. 2018; 2018: bay018.

[2] Zhu H. Editorial for the Special Issue Challenges in Computational Neuroscience. Statistics in Biosciences. 2019; 11: 1-2.

[3] Goñi J, Esteban FJ, de Mendizábal N, Sepulcre J, ArdanzaTrevijano S, Agirrezabal I, et al. A computational analysis of protein-protein interaction networks in neurodegenerative diseases. BMC Systems Biology. 2008; 2: 52.

[4] Taguchi Y, Nishida N. Secondary-structure prediction revisited: Theoretical $\beta$-sheet propensity and coil propensity represent structures of amyloids and aid in elucidating phenomena involved in interspecies transmission of prions. PLoS ONE. 2017; 12: e0171974.

[5] Jha NK, Kar R, Niranjan R. ABC Transporters in Neurological Disorders: an Important Gateway for Botanical Compounds Mediated Neuro-Therapeutics. Current Topics in Medicinal Chemistry. 2019; 19: 795-811.

[6] Jha NK, Jha SK, Sharma R, Kumar D, Ambasta RK, Kumar P. Hypoxia-Induced Signaling Activation in Neurodegenerative Diseases: Targets for New Therapeutic Strategies. Journal of Alzheimer's Disease. 2018; 62: 15-38.

[7] Jha SK, Jha NK, Kumar D, Sharma R, Shrivastava A, Ambasta RK, et al. Stress-Induced Synaptic Dysfunction and Neurotransmitter Release in Alzheimer's Disease: can Neurotransmitters and Neuromodulators be Potential Therapeutic Targets? Journal of Alzheimer's Disease. 2017; 57: 1017-1039.

[8] Sharma R, Kumar D, Jha NK, Jha SK, Ambasta RK, Kumar P. Re-expression of cell cycle markers in aged neurons and muscles: whether cells should divide or die? Biochimica Et Biophysica Acta (BBA)-Molecular Basis of Disease. 2017; 1863: 324-336.

[9] Jha NK, Jha SK, Kar R, Nand P, Swati K, Goswami VK. Nuclear factor-kappa $\beta$ as a therapeutic target for Alzheimer's disease. Journal of Neurochemistry. 2019; 150: 113-137.
[10] Jha SK, Jha NK, Kar R, Ambasta RK, Kumar P. P38 MAPK and PI3K/AKT Signalling Cascades in Parkinson's Disease. International Journal of Molecular and Cellular Medicine. 2015; 4: 67-86.

[11] Jha NK, Jha SK, Kar R, Ambasta RK, Kumar P. Role of oxidative stress, ER stress and Ubiquitin Proteasome system in neurodegeneration. MOJ Cell Science and Report. 2014; 1: 38-44.

[12] Jha SK, Jha NK, Kumar D, Ambasta RK, Kumar P. Linking mitochondrial dysfunction, metabolic syndrome and stress signaling in Neurodegeneration. Biochimica et Biophysica Acta. Molecular Basis of Disease. 2017; 1863: 1132-1146.

[13] Kumar P, Jha NK, Jha SK, Ramani K, Ambasta RK. Tau phosphorylation, molecular chaperones, and ubiquitin E3 ligase: clinical relevance in Alzheimer's disease. Journal of Alzheimer's Disease. 2015; 43: 341-361.

[14] Jha NK, Jha SK, Kumar D, Kejriwal N, Sharma R, Ambasta RK, et al. Impact of Insulin Degrading Enzyme and Neprilysin in Alzheimer's Disease Biology: Characterization of Putative Cognates for Therapeutic Applications. Journal of Alzheimer's Disease. 2015; 48: 891-917.

[15] Cho DY, Kim YA, Przytycka TM. Chapter 5: Network biology approach to complex diseases. PLOS Computational Biology. 2012; 8: e1002820.

[16] Kriegeskorte N, Douglas PK. Cognitive computational neuroscience. Nature Neuroscience. 2018; 21: 1148-1160.

[17] Nayak L, Dasgupta A, Das R, Ghosh K, De RK. Computational neuroscience and neuroinformatics: Recent progress and resources. Journal of Biosciences. 2018; 43: 1037-1054.

[18] Vasaikar SV, Padhi AK, Jayaram B, Gomes J. NeuroDNet - an open source platform for constructing and analyzing neurodegenerative disease networks. BMC Neuroscience. 2013; 14: 3.

[19] Segovia F, Górriz JM, Ramírez J, Salas-Gonzalez D, Álvarez I, López M, et al. A comparative study of feature extraction methods for the diagnosis of Alzheimer's disease using the ADNI database. Neurocomputing. 2012; 75: 64-71.

[20] Spering CC, Hobson V, Lucas JA, Menon CV, Hall JR, O’Bryant SE. Diagnostic accuracy of the MMSE in detecting probable and possible Alzheimer's disease in ethnically diverse highly educated individuals: an analysis of the NACC database. Journals of Gerontology. Series a, Biological Sciences and Medical Sciences. 2012; 67: 890-896.

[21] Keane H, Ryan BJ, Jackson B, Whitmore A, Wade-Martins R Protein-protein interaction networks identify targets which rescue the MPP+ cellular model of Parkinson's disease. Scientific Reports. 2015; 5: 17004

[22] Tanabe M, Limviphuvadh V, Kanehisa M. Knowledge-Based Analysis of Protein Interaction Networks in Neurodegenerative Diseases. Neuroproteomics. 2010; 5: 147-162.

[23] Nguyen T, Caberlotto L, Morine MJ, Priami C. Network analysis of neurodegenerative disease highlights a role of Toll-like receptor signaling. BioMed Research International. 2014; 2014: 686505.

[24] Eberini I, Daniele S, Parravicini C, Sensi C, Trincavelli ML, Martini C, et al. In silico identification of new ligands for GPR17: a promising therapeutic target for neurodegenerative diseases. Journal of Computer-Aided Molecular Design. 2011; 25: 743-752.

[25] Li P, Nie Y, Yu J. An Effective Method to Identify Shared Pathways and Common Factors among Neurodegenerative Diseases. PLoS ONE. 2015; 10: e0143045.

[26] Casas C, Isus L, Herrando-Grabulosa M, Mancuso FM, Borrás E, Sabidó E, et al. Network-based proteomic approaches reveal the neurodegenerative, neuroprotective and pain-related mechanisms involved after retrograde axonal damage. Scientific Reports. 2015; 5: 9185.

[27] Brown KR, Jurisica I. Online predicted human interaction database. Bioinformatics. 2005; 21: 2076-2082.

[28] Cesareni G, Chatr-aryamontri A, Licata L, Ceol A. Searching the MINT database for protein interaction information. Current Protocols in Bioinformatics. 2008; Chapter 8: Unit 8.5.

[29] Srinivasa Rao V, Srinivas K, Kumar GN, Sujin GN. Protein inter- 
action network for Alzheimer's disease using computational approach. Bioinformation. 2013; 9: 968-972.

[30] Panda A, Begum T, Ghosh TC. Insights into the evolutionary features of human neurodegenerative diseases. PLoS ONE. 2012; 7: e48336.

[31] Mehra R, Kepp KP. Computational analysis of Alzheimer-causing mutations in amyloid precursor protein and presenilin 1. Archives of Biochemistry and Biophysics. 2019; 678: 108168.

[32] Monteiro AFM, Viana JDO, Nayarisseri A, Zondegoumba EN, Mendonça Junior FJB, Scotti MT, et al. Computational Studies Applied to Flavonoids against Alzheimer's and Parkinson's Diseases. Oxidative Medicine and Cellular Longevity. 2018; 2018: 7912765.

[33] Pinhiero AA, Silva KRd, Silva AES, Braga FS, Silva CHTPd, Santos CBR, et al. In silico identification of novel potential BACE-1 inhibitors for Alzheimer's disease treatment: Molecular docking, pharmacophore modeling and activity and synthetic accessibility predictions. British Journal of Pharmaceutical Research. 2015; 7: 217-229.

[34] Nataf S, Guillen M, Pays L. Common NeurodegenerationAssociated Proteins Are Physiologically Expressed by Human B Lymphocytes and Are Interconnected via the Inflammation/Autophagy-Related Proteins TRAF6 and SQSTM1. Frontiers in immunology. 2019; 10: 2704.

[35] Wroe R, Wai-Ling Butler A, Andersen PM, Powell JF, Al-Chalabi A. ALSOD: the Amyotrophic Lateral Sclerosis Online Database. Amyotrophic Lateral Sclerosis. 2008; 9: 249-250.

[36] Ahmad K, Baig MH, Mushtaq G, Kamal MA, Greig NH, Choi I. Commonalities in Biological Pathways, Genetics, and Cellular Mechanism between Alzheimer Disease and other Neurodegenerative Diseases: an in Silico-Updated Overview. Current Alzheimer Research. 2017; 14: 1190-1197.

[37] Rajasekaran S, Chitraa T, Dilip Chand Raja S, Raveendran M, Sharon Miracle N, Sri Vijayanand KS, et al. Subclinical infection can be an initiator of inflammaging leading to degenerative disk disease: evidence from host-defense response mechanisms. European Spine Journal. 2021. (in press)

[38] Martin-Martin A, Orduna-Malea E, Harzing A, Delgado LópezCózar E. Can we use Google Scholar to identify highly-cited documents? Journal of Informetrics. 2017; 11: 152-163.

[39] Fernandopulle MS, Lippincott-Schwartz J, Ward ME. RNA transport and local translation in neurodevelopmental and neurodegenerative disease. Nature Neuroscience. 2021; 24: 622-632.

[40] Miryala SK, Anbarasu A, Ramaiah S. Discerning molecular interactions: a comprehensive review on biomolecular interaction databases and network analysis tools. Gene. 2018; 642: 84-94.

[41] Haraldstad AB, Christophersen E. Literature Searches and Reference Management. Research in Medical and Biological Sciences. 2015; 281: 125-165.

[42] Gowthaman R, Gowthaman N, Rajangam MK, Srinivasan K. Database of neurodegenerative disorders. Bioinformation. 2007; 2: 153-154.

[43] Na D, Rouf M, O'Kane CJ, Rubinsztein DC, Gsponer J. Neuro-
GeM, a knowledgebase of genetic modifiers in neurodegenerative diseases. BMC Medical Genomics. 2013; 6: 52.

[44] Crespo I, Roomp K, Jurkowski W, Kitano H, del Sol A. Gene regulatory network analysis supports inflammation as a key neurodegeneration process in prion disease. BMC Systems Biology. 2012; 6: 132 .

[45] Dovrolis N, Kolios G, Spyrou G, Maroulakou I. Laying in silico pipelines for drug repositioning: a paradigm in ensemble analysis for neurodegenerative diseases. Drug Discovery Today. 2017; 22: 805-813.

[46] Pathak A, Madar IH, Raithatha K, Gupta JK, In-Silico Identification of Potential Inhibitors Against AChE Using Cheminformatics Approach. MOJ Proteomics \& Bioinformatics. 2014; 1: 1-5.

[47] Rakshit H, Rathi N, Roy D. Construction and analysis of the protein-protein interaction networks based on gene expression profiles of Parkinson's disease. PLoS ONE. 2014; 9: e103047.

[48] Godini R, Fallahi H, Ebrahimie E. A comparative system-level analysis of the neurodegenerative diseases. Journal of Cellular Physiology. 2019; 234: 5215-5229.

[49] Karbalaei R, Allahyari M, Rezaei-Tavirani M, Asadzadeh-Aghdaei $\mathrm{H}$, Zali MR. Protein-protein interaction analysis of Alzheimer's disease and NAFLD based on systems biology methods unhide common ancestor pathways. Gastroenterology and Hepatology from Bed to Bench. 2018; 11: 27-33.

[50] Hallock P, Thomas MA. Integrating the Alzheimer's disease proteome and transcriptome: a comprehensive network model of a complex disease. Omics. 2012; 16: 37-49.

[51] Winkler JM, Fox HS. Transcriptome meta-analysis reveals a central role for sex steroids in the degeneration of hippocampal neurons in Alzheimer's disease. BMC Systems Biology. 2013; 7: 51.

[52] Han Z, Huang H, Gao Y, Huang Q. Functional annotation of Alzheimer's disease associated loci revealed by GWASs. PLoS ONE. 2017; 12: e0179677.

[53] George G, Singh S, Lokappa SB, Varkey J. Gene co-expression network analysis for identifying genetic markers in Parkinson's disease-a three-way comparative approach. Genomics. 2019; 111: 819-830.

[54] Barh D, García-Solano ME, Tiwari S, Bhattacharya A, Jain $\mathrm{N}$, Torres-Moreno D, et al. BARHL1 is Downregulated in Alzheimer's Disease and may Regulate Cognitive Functions through ESR1 and Multiple Pathways. Genes. 2017; 8: 245.

[55] Hassan M, Shamas M, Khalil M, El Falou W, Wendling F. EEGNET: an Open Source Tool for Analyzing and Visualizing M/EEG Connectome. PLoS ONE. 2015; 10: e0138297.

[56] Chin C, Chen S, Wu H, Ho C, Ko M, Lin C. CytoHubba: identifying hub objects and sub-networks from complex interactome. BMC Systems Biology. 2014; 8: S11.

[57] Wang X, Hu H, Huang C, Kennedy H, Li CT, Logothetis N, et al. Computational neuroscience: a frontier of the 21 st century. $\mathrm{Na}$ tional Science Review. 2020; 7: 1418-1422.

[58] Hassabis D, Kumaran D, Summerfield C, Botvinick M. Neuroscience-Inspired Artificial Intelligence. Neuron. 2017; 95: 245-258. 